\title{
Hedgehog signalling network gene status analysis in paediatric intracranial germ cell tumours
}

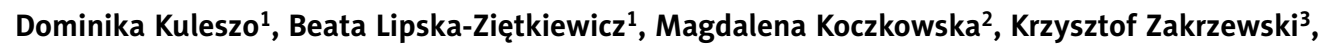 \\ Wiesława Grajkowska ${ }^{4}$, Marcin Roszkowski ${ }^{5}$, Bożenna Dembowska-Bagińska ${ }^{6}$, Katarzyna Czarnota ${ }^{7}$, \\ Elżbieta Adamkiewicz-Drożyńska ${ }^{8}$, Ewa lżycka-Świeszewska9
}

${ }^{1}$ Clinical Genetics Unit, Department of Biology and Medical Genetics, Medical University of Gdańsk, Poland, ${ }^{2}$ Department of Biology and Medical Genetics, Medical University of Gdańsk, Poland, ${ }^{3}$ Department of Neurosurgery, Polish Mother's Memorial Hospital Research Institute, tódź, Poland, ${ }^{4}$ Department of Pathology, Children's Health Memorial Institute, Warsaw, Poland, ${ }^{5}$ Department of Neurosurgery, Children's Health Memorial Institute, Warsaw, Poland, ${ }^{6}$ Department of Oncology, Children's Health Memorial Institute, Warsaw, Poland, ${ }^{7}$ Department of Pathomorphology, Copernicus Hospitals, Gdańsk, Poland, ${ }^{8}$ Department of Paediatrics, Oncology and Haematology, Medical University of Gdańsk, Poland, ${ }^{9}$ Department of Pathology and Neuropathology, Medical University of Gdańsk, Poland

\begin{abstract}
Introduction: Germ cell tumours (GCTS) in the children comprise a group of tumours that originate from primordial germ cells but their pathogenesis is not clear. Intracranial GCTs represent a special subset of these paediatric neoplasms. Hedgehog (Hh) pathway gene status in GCTs is generally unexplored, while Hh signalling is involved in germ cell biology.

Material and methods: Comparative genomic profiling analysis with a microarray-comparative genomic hybridization $(C G H)+$ single nucleotide polymorphism (SNP) technique in a group of intracranial paediatric GCTs was performed. The analysis included evaluation of genes being ligands, receptors, regulators, effectors, and targets of Hh signalling. Results: Chromosomal aberrations were found in 62\% of examined tumours, showing their heterogeneity. A number of private genomic imbalances were observed, but only a few recurrent ones. The most common numerical changes were trisomies 19, 21 and monosomies 13, 18 while the most frequent structural aberration was gain/ amplification of the chromosome 12p. The analysis of the gene status of Hh network elements showed imbalances in a proportion of tumours. PTCH1, GLI2, IHH and ZIC2 gene aberrations occurred most frequently. Moreover, six tumours had various copy gains or losses of several other genes involved in the pathway, including HHIP, GLII, GLI3, DHH, SHH, SMO, PTCH2, and several genes from the WNT group. Interestingly, four cases showed losses of pathway repressors, with parallel gains of activators in two of them. Correlations with patho-clinical tumour features were not found, most probably due to the heterogeneity of the examined limited group.

Conclusions: Our results show few genomic alterations related to the Hh signalling pathway genes in paediatric intracranial GCTs. Further analysis of Hedgehog pathway alterations can potentially disclose its biological significance and define new prognostic factors and/or therapeutic targets for high-risk patients.
\end{abstract}

Key words: genomic imbalances, intracranial germ-cell tumours, PTCH1, Hedgehog signalling, ZIC2.

\section{Communicating author}

Prof. Ewa Iżycka-Świeszewska, Department of Pathology and Neuropathology, Medical University of Gdańsk, 1 Dębinki St., 80-211 Gdańsk, Poland, e-mail: eczis@gumed.edu.pl 


\section{Introduction}

Germ cell tumours (GCTs) encompass a group of neoplasms with a diverse clinical picture depending on the patients' age and tumour location. GCTs are classified into several histological subtypes and in a significant portion of cases present as mixed tumours. The vast majority of these tumours are located in gonads, while extragonadal cases develop in the mediastinum, retroperitoneum, sacral region, and in the central nervous system (CNS) [34,39]. GCTs arising in the CNS affect mainly adolescent boys and young men and grow in the pineal, suprasellar region and around the third ventricle. They constitute $0.5 \%$ of all intracranial neoplasms, with unexplained 3-5 fold higher incidence in Asians than in other ethnicities. The most common type of CNS GCT is germinoma; teratomas, yolk sac or mixed tumours are less frequently encountered [14,15].

Paediatric GCTs are rare and very special neoplasms with dedicated therapeutic approaches for different patho-clinical subtypes $[15,39]$. Their treatment remains a challenge due to their substantial biological heterogeneity resulting in variable clinical behaviour and prognosis. GCTs are managed with various combinations of surgery, chemotherapy and radiotherapy with special dedicated protocols for intracranial GCTs. CNS germinomas are very sensitive to radio- and chemotherapy with long-term survival rates. However, CNS irradiation in young age is associated with side effects and decreased quality of life afterwards. The non-germinomatous malignant tumours vary in therapeutic response resulting in a less satisfactory outcome $[5,22,39]$. There is a need to create new, more effective therapeutic strategies with lower toxicity, which might be possible through a better understanding of the biological mechanisms of development and progression of these neoplasms.

Several distinct pathogenetic theories on GCTs' origin have been proposed. In general, paediatric GCTs are considered to come from the primordial germ cells at different stages of differentiation. The less popular theory of organ-specific tissue stem cells was also postulated for the CNS lesions. The pathogenesis of GCTs is explained as being a result of disturbances of cell division, inhibition of apoptosis, aberrations of various signalling pathways, changes in gene expression and disrupted migration of embryonic cells $[42,43,48]$. Recent integrated analyses on adult testicular tumours identified distinct molecular patterns that characterized histologic subtypes and revealed the important role of epigenetic processes in determining histologic and biologic features [46]. However, it seems that paediatric GCTs located in different sites arise through diverse mechanisms as a result of a cross-talk between maldevelopmental and oncogenic events. Increasing amounts of data indicate the specific role of several signal transduction pathways in the biology of germ cells. One of them, explored to a much lesser extent than others, is the hedgehog $(\mathrm{Hh})$ signalling $[11,41]$.

The hedgehog pathway affects many developmental processes controlling cell migration, proliferation and differentiation as well as intercellular communication. The $\mathrm{HH}$ network plays a crucial role in organogenesis and maintenance of stem cell populations as well as reparative and regenerative processes [37]. To date, the best-studied functions of the pathway have been the cell type specification and patterning of the central nervous system, axial formation of the embryo as well as limb and tooth development [51]. This pathway was originally discovered in Drosophila melanogaster, but a breakthrough in understanding the link between Hh signalling and carcinogenesis came from the Gorlin syndrome (also: nevoid basal cell carcinoma syndrome-NBCCS; MIM:\#109400) [33]. This syndrome, caused by mutations in the PTCH1 gene, predisposes to basal cell carcinoma, different benign and malignant tumours, and some skeletal abnormalities $[1,6,16]$. The cancerogenic Hh signalling also occurs in many sporadic malignant tumours including gastro-intestinal, haematological, breast, prostate, lung, brain and skin cancers $[3,26,35,38]$. Hh alterations in paediatric cancer concern a proportion of medulloblastomas (Hh-activated molecular subtype), some rhabdomyosarcomas, neuroblastomas and several others $[16,18,26,29,40]$. The oncogenic signalling stimulates proliferation and survival of cancer stem cells, affects their differentiation, controls angiogenesis and tumour-stroma interactions. Currently, several agents targeting overactivated elements of the hedgehog pathway (e.g. $\mathrm{HH}, \mathrm{SMO}, \mathrm{GLI}$ ) are under development and have recently been tested in clinical trials on different tumours, some of them showing promising results $[17,26]$.

Genetic analyses performed on adult onset testicular GCTs allowed to identify several distinct cytogenetic patterns. Little is known about molecular 
profiles of paediatric extragonadal tumours. Here, we evaluated the genomic imbalances in paediatric intracranial germ cell tumours with a special scope on the loci of genes involved in the Hedgehog pathway as a new direction of their investigation.

\section{Material and methods \\ Tumour specimens}

In total, 16 unrelated patients with intracranial tumours were enrolled in this study, including five previously reported in our pilot study [25]. The clinicopathological data completely anonymized the patients' age, gender, tumour histology, diameter and site of development, as well as therapeutic methods and information on survival (data of the analysed cases are presented in Table I). Patients were recruited from two paediatric centres in Poland. None of the patients were previously treated oncologically. In six cases of deep seated tumours, only stereotactic biopsy was performed. Each of the studied tumours underwent an intraoperative exam and fresh-frozen tissues were collected for molecular analysis. In the final pathological exam, immunohistochemistry was applied for the differential diagnosis. The panel of antibodies included organic cation transporter 3 (Oct3), placental alkaline phosphatase (PLAP), $\alpha$-fetoprotein (AFP), CD30, CD117, cytokeratin AE1/AE3, cytokeratin 7, glypican 3, endomysial antibody (EMA), leukocyte common antigen (LCA), and anti-glial fibrillary acidic protein (GFAP). All procedures were carried out according to the manufacturer's recommendations in a DAKO autostainer. In each case the second pathological opinion, based on routinely processed tumour sections, was performed in an external centre.

In three cases (germinomas \#4 and \#11, and teratoma \#1), immunohistochemistry with $\mathrm{SHH}$ and PTCH1 antibodies (Abcam) was also performed on paraffin-embedded tissue samples with a standard procedure based on DAKO reagents.

\section{DNA extraction}

Fresh-frozen tumour tissue samples from intracranial surgery $(n=10)$ or stereotactic biopsy $(n=6)$ were collected and subjected to DNA extraction using the salting-out protocol adapted after Miller et al. [31]. Based on the obtained DNA quality and quantity, measured by both spectrophotometric and fluorometric methods on NanoDrop ND-100 (Thermo-
Fisher) and Qubit 2.0 Fluorometer dsDNA BR Assay (ThermoFisher) respectively, 14/16 tumours were qualified for genomic profiling.

\section{Genomic profiling (array-CGH analysis)}

Array comparative genomic hybridization (array$\mathrm{CGH})$ at the average resolution of $150 \mathrm{~kb}$ was performed using SurePrint G3 Cancer CGH+SNP Microarray Kit, 4x180K (Agilent Technologies) - cases \#1-8, and Cytosure ISCA UPD 4x180K (Oxford Gene Technology) - cases \#9-13. As the reference control DNA, sex-mismatched Human Genomic DNA (Promega) was used. Arrays were scanned at $2 \mu \mathrm{m}$ resolution (MS200 Microarray Scanner; Roche). Simultaneous evaluation of copy number alterations and changes in heterozygosity was performed using CytoGenomics (Agilent Technologies) and Nexus Copy Number 8.0 (BioDiscovery) programs. All identified genomic imbalances were verified against the online Database of Genomic Variants (DGV; last accessed in May 2018) [28] and the in-house database of $>1000$ benign copy number variations (CNVs) identified in the local population. Numbering of map positions was based on hg19/GRCh37 reference sequence.

The 84 genes chosen for the analysis of the Hedgehog pathway and its network were based on a set of genes included in the commercial kit designed by Qiagen for Hh signalling gene expression analysis (Hedgehog Signalling Pathway RT ${ }^{2}$ Profiler PCR Array, Qiagen, prod. no: PAHS-078Z).

\section{Results}

Of the 16 cases enrolled in the study, genomic profiles were analysed for 13 tumours, as two cases had unsatisfactory DNA extraction quality and one sample failed array-CGH quality control threshold due to the high derivative log ratio spread. The patho-clinical characteristics are summarized in Figure 1 and Table I.

Application of the CGH + SNP technique allowed simultaneous analysis of copy number changes and heterozygosity alterations leading to much better resolution and characterization of the nature of the observed changes. That included a re-evaluation of the five cases published in our previous pilot study [25], revealing additional data: three recurrent genomic imbalances in one case initially classified as diploid and presence of additional copy number variations (CNVs) in another two cases. 


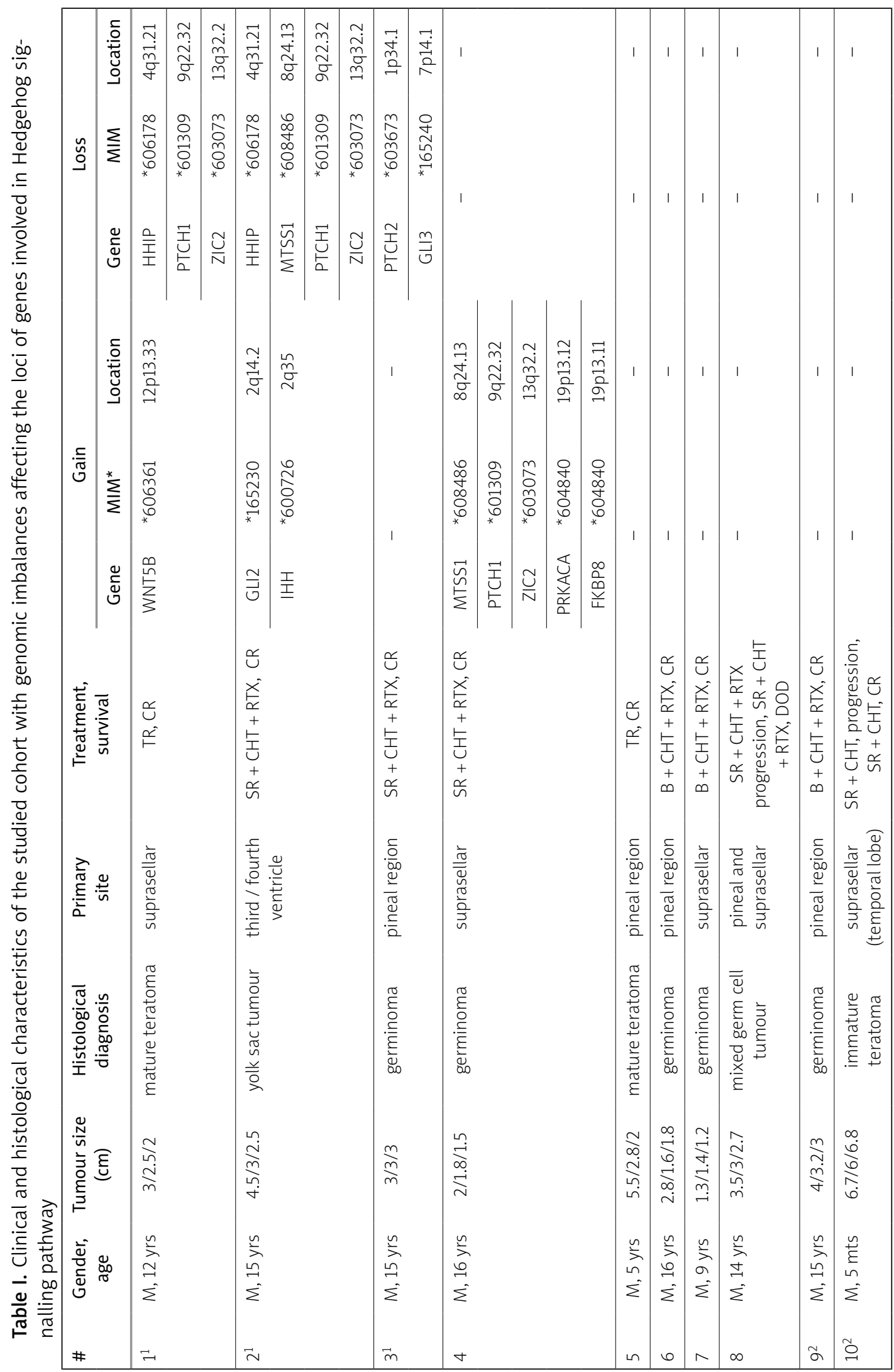




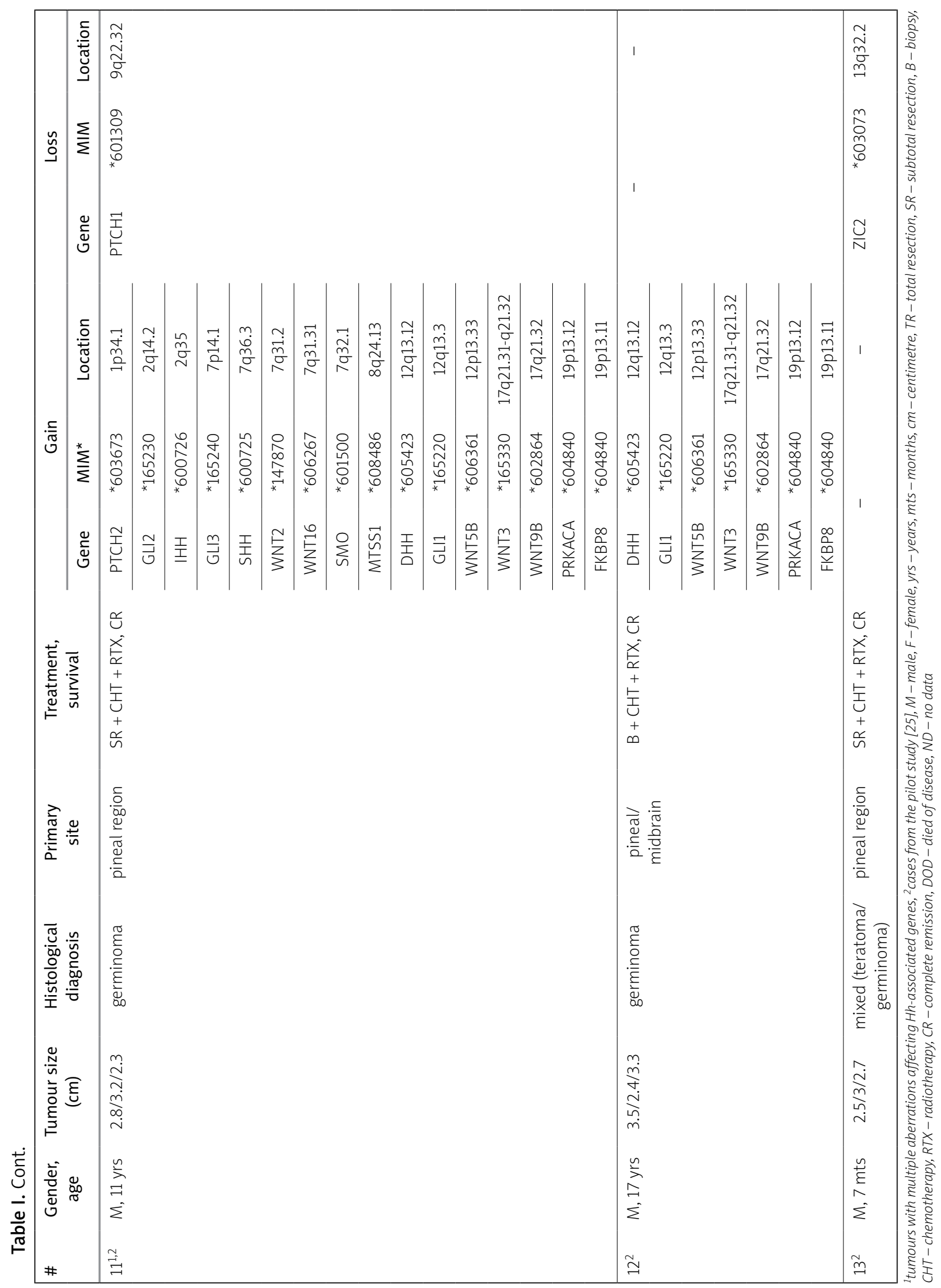



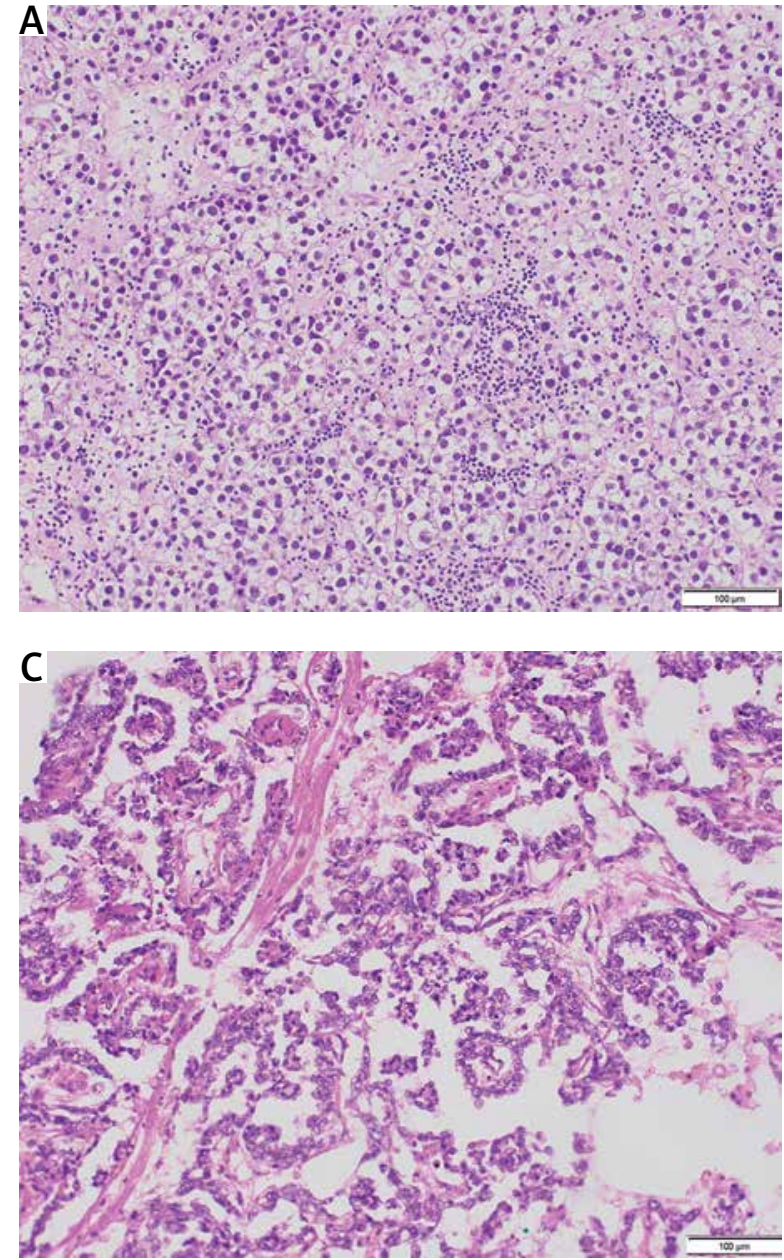

\section{Genomic imbalances}

Genomic imbalances were revealed in 8/13 GCTs $(62 \%)$, while the remaining five tumours exhibited normal genomic profiles with a few relatively small $(<300 \mathrm{~kb})$ benign recurrent CNVs. Detailed genomic profiles of all the tested tumours are available upon request.

Chromosomes 1, 2, 5, 6, 7, 8, 9, 13, 14, 15, 19, 20, $21,22, X$ and $Y$ were involved in gains of genetic material, while chromosomes $4,5,6,9,13,15,16$, 18 and 22 were involved in losses. The sole presence of duplications and/or amplifications was detected in 2/13 tumours, while loss of genetic material was exclusively identified in a single tumour. CNAs affecting chromosomes 14, 19, 20 and 21 were gains only, while chromosomes 4 and 11 were only affected by deletions. Complex genomic profiles did not involve chromosome 3 in any case. Two cases: \#4 and \#11 were classified as near-triploid.

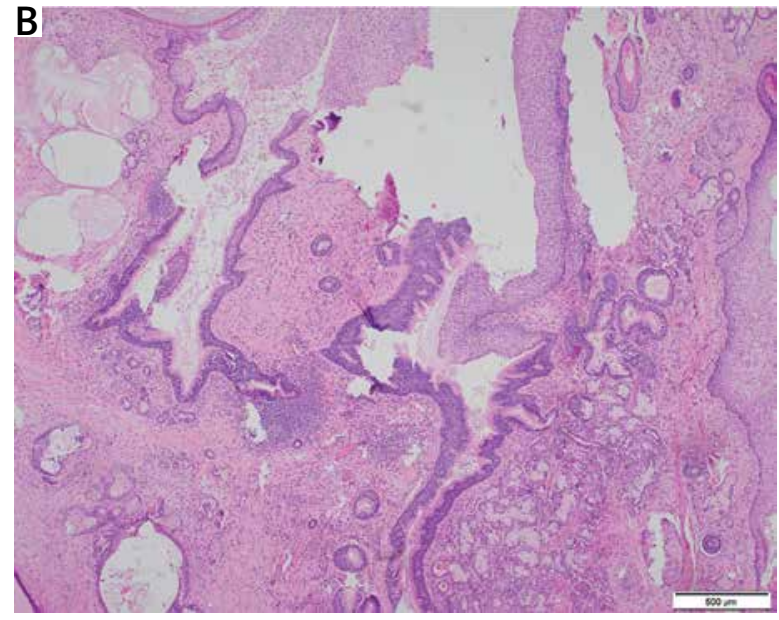

Fig. 1. Histopathologic features of germ cell tumours: A) Germinoma composed of large roundish cells with clear cytoplasm, big hyperchromatic nuclei and prominent nucleoli. There is a prominent stromal lymphocytic infiltration (HE, 200x, 400x); B) Mature teratoma consisting of mature tissues originating from all three germ layers - endoderm i.e. gastrointestinal and respiratory epithelium, mesoderm (e.g. cartilage, muscle fibres) and ectoderm (i.e. epidermis and adnexa) (HE, 40x, 100x); C) Yolk sac tumour made of cells arranged in a cystic and reticular pattern with characteristic glomeruloid perivascular structures called Shiller-Duvall bodies (HE, $200 \times, 400 \times)$.

\section{Numerical aberrations}

The most frequent numerical aberration was trisomy of chromosome 21, detected in 31\% (4/13) of cases including two mature teratomas (\#1 and \#5), one germinoma (\#11) and one mixed tumour composed of teratoma and germinoma components (\#13). In two cases it was the only gain observed (\#5, \#13). In fact, one of the boys affected with one of these tumours had been diagnosed with Down syndrome (\#1). Another recurrent genomic imbalance was trisomy of chromosome 19, containing loci to FKBP8 (MIM: *604840) and PRKACA (MIM: *604840) (involved in $\mathrm{Hh}$ network) present in $75 \%$ (3/4) of the analysed cases of germinoma tumours with an abnormal molecular karyotype. The most frequently observed losses were monosomy of chromosomes 13 (encompassing among others loci of FGF9 and ZIC2) and 18 (including BCL2 and NPC1 loci), occurring simultaneously in three distinct histological subtypes: 
a mature teratoma (\#1), a yolk sac tumour (\#2) and a mixed tumour with teratoma and germinoma components (\#13). Additionally, a loss of the long arm of chromosome 18 was present in one case (\#12).

\section{Structural aberrations and Hh pathway genomic loci}

Structural abnormalities involving fragments of chromosomes 1, 2, 6, 7, 8, 9, 10, 11, 12, 16, 17 and 22 were present in $6 / 13$ tumours. Five chromosomal regions were altered in more than one case: two partial gains and three partial losses of chromosomes 12, 17 and 6, 9, 11 respectively. The most frequently affected chromosomal region was the short arm of chromosome 12 altered in 3/13 tumours (23\%). One mature teratoma (\#1) and one germinoma (\#11) presented amplification of the short arm of chromosomes 12 (12p13.33p11.1), involving KRAS (MIM: $\left.{ }^{*} 190070\right)$ at $12 \mathrm{p} 12.1$ and WNT5B (MIM: $\left.{ }^{*} 606361\right)$ at 12 p13.33 loci, while the gain of $12 p$ was identified in an additional germinoma tumour (\#12). The gain encompassing the long arm of chromosome 17 with a minimal overlapping region at $17 q 11.1 q 25.3$ was present in two germinoma tumours (\#11 and \#12). Also, gain of 17q25.3 fragment was detected in a yolk sac tumour (\#2). Among losses, deletion of 6q12-q27 region in suprasellar mature teratoma (\#1) and germinoma located in the pineal region (\#3) with a minimal overlapping region of $103.8 \mathrm{Mb}$, was noted. Additionally, a partial deletion of the chromosome 9 (9q21.11-q34.4) encompassing loci of Hh pathway iconic receptor gene $P T C H 1$ regulating $G A S 1$ and networking FOXE 1 was observed in a yolk sac tumour (\#2) and a pineal germinoma (\#11). Deletion of the short arm of chromosome 11 (11p15.5-p11.12) was detected in a yolk sac tumour (\#2) and a midbrain germinoma (\#12). Also a deletion of the $11 \mathrm{q}$ region was detected in yolk sac tumour (nullisomy) and \#11 germinoma, with minimal overlapping region $11 \mathrm{q} 13.5-\mathrm{q} 25$.

Analysis of the genomic loci encompassing genes coding for ligands, receptors, regulators, cofactors and target genes of the mainstream Hedgehog signalling pathway in all analysed cases revealed several changes (Table I). The alterations of other genes cooperating with $\mathrm{Hh}$ signalling were also detected (a comprehensive list of the analysed genes is available upon request).

The aberrations included both gains and losses of genetic material covering genes associated with $\mathrm{Hh}$ signalling transduction. The most common changes affected PTCH1 (MIM: *601309) and ZIC2 (MIM: *603073) genes. The simultaneous loss of PTCH1, ZIC2 and HHIP (MIM: *606178) genes was reported in two tumours: a mature teratoma (\#1) and a yolk sac tumour (\#2). Besides, a sole loss of PTCH1 or ZIC2 gene was observed in a pineal germinoma (\#11) and a teratoma with germinoma component (\#13) respectively. Conversely, additional copies of $\mathrm{PTCH} 1$ and ZIC2 were found in a single suprasellar germinoma (\#4). A gain of the GLI2 (MIM: $\left.{ }^{*} 165230\right)$ and IHH (MIM: *600726) genes, both located on chromosome 2 in a yolk sac tumour (\#2) and a pineal gland germinoma (\#11) were detected. Other changes, involving HHIP, GLI1, GLI3, DHH, SHH, SMO, PTCH2 and PTCHD occurred sporadically (Table I). Moreover, genomic instability affected loci of FGFR3, FGF9, LATS1, STK3 and numerous members of BMPS and WNTs gene families.

Relations between the observed alterations and tumour histology or patients' age were not revealed.

Finally, immunohistochemistry, performed to assess protein expression, showed strong positive cytoplasmic labelling with $\mathrm{SHH}$ antibody in germinomas and heterogenous histological structure-dependent staining in teratoma. PTCH1 expression was found as strong nuclear in germinomas and membranous, cytoplasmic or nuclear in different histological elements of teratomas (Fig. 2).

\section{Discussion}

Growing evidence demonstrates that embryogenesis and cancerogenesis have common features and executive pathways. Paediatric GCTs, which often have midline localization and present multidirectional differentiation, are believed to develop from the primordial germ cells. The primordial germ cells are specified from the non-germline lines by genetic and epigenetic mechanisms, maintaining pluripotency and proliferative potential. They migrate along the body to locate in the primitive gonads under control of complex mechanisms involving, among other things, sterol concentrations and Hh guiding. Further gonadal development is also connected to this pathway $[4,11,12,20,34,38]$.

$\mathrm{Hh}$ signalling is a well-established, important regulator of cell migration and cell type specification in development, regeneration, reparation, and neoplasia [3,20,37]. Abnormal Hh pathway function observed in cancer has become a field of intensive 

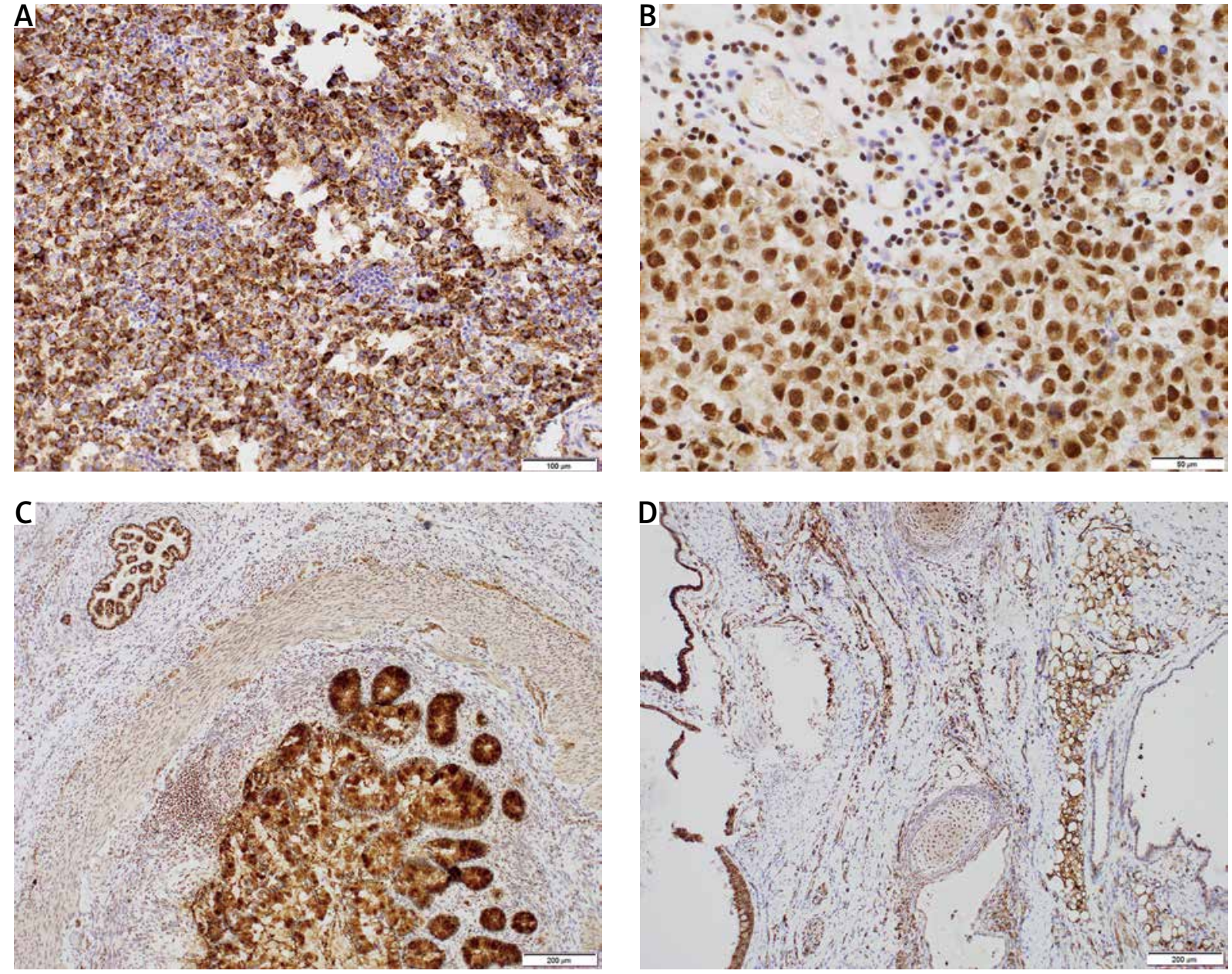

Fig. 2. Immunohistochemical staining: A) Germinoma - diffuse intense reactivity for SHH in neoplastic cells, stromal lymphocytes are negative (SHH 200x); B) Germinoma - intense nuclear expression of PTCH1 within the neoplastic cells, stromal cells are negative (PTCH1, 400x); C, D) Mature teratoma disclosing different reactivity of examined proteins in relation to tissue histology. Heterogenous PTCH1 labelling within the cytoplasm/membrane, and nuclear in some cells. SHH cytoplasmic staining with varied intensity (PTCH1 40x, SHH 100x).

research in the context of basic science and targeted oncological therapy $[4,20]$. Activation of the Hh pathway may occur in canonical, ligand-dependent $\mathrm{SHH}$, $\mathrm{DHH}, \mathrm{IHH}$ ) interaction or through receptor-induced stimulation and non-canonical, ligand-independent mode. The source of $\mathrm{HH}$ ligand can be cancer cells (autocrine and paracrine stimulation), tumour microenvironment (reverse paracrine stimulation), as well as peritumoral tissue secretion. In canonical activation, an interaction between the ligand and membrane receptor complex Patched 1 (PTCH1) and Smoothened (SMO) occurs. Activated SMO triggers downstream cascade leading to nuclear translocation and activation of GLI transcription factor family or other target genes. GLI1 works as a transcription activator, while GLI2 and GLI3 can act as activators or repressors. GLI1-3 are powerful transcription factors having many target genes and complicated crosstalk. Hedgehog signalling cascade undergoes regulation by SUFU, HHIP, GPC3 and RAB23 proteins $[3,27]$. The aberrant activation in cancer may be caused also by mutations in genes such as $P T C H$, SUFU, SMO, and GLI. Furthermore, signalling can be executed by disequilibrium of activating and inhibiting factors. These include overexpression of SMO, GLI1 and other regulatory enhancers or decreased/ silenced expression or aberrant function of PTCH1, SUFU, and cooperating repressors. Important in 
oncogenic signalling are crosstalks with transforming growth factor $\beta$ (TGF- $\beta$ )/BMP, WNT, Hippo, EGFR, K-Ras, PKA, Notch, PKC, Mek1, GSK3B, PI3K and other systems $[19,35,36]$. Therefore, a whole-genome screening approach with using the microarray-CGH technique was applied in the current report to evaluate the signs of alterations at various points of the pathway in the examined group of intracranial paediatric GCTs. Our preliminary data [25] were subsequently revised and extended by an analysis of an additional series of these rare tumours.

Despite the limited number of cases analysed herein, the array-CGH analysis of genomic profiles of a series of CNS GCTs provides new results on the Hh status. Only half of the germinomas studied by us presented genomic imbalances, while the other histological subtypes showed a wide range of chromosomal aberrations, both numerical and structural. Two out of four germinomas with an aberrant genomic profile most likely were simply triploid, while the other types of GCTs had specific chromosomal changes. In the previous studies, the number of CNS GCTs with genomic imbalances was around $90 \%[44,45,50]$. The observed discrepancies might have resulted from either: 1) solely paediatric population of patients analysed in the current study since tumours in children ( $<18$ years of age) constituted only $58-74 \%$ in the previous studies $[44,45,50]$; 2) minimal content of tumour cells in some of the analysed specimens; and 3) different methodology and criteria used. The normal genomic profiles of some of the examined cases could be caused by poor representativeness of frozen samples due to stromal lymphocytic infiltrates in scanty material. Finally, this report contains only the imbalances present reliably in the largest part of each specimen analysed. Conversely, the virtual karyotyping (NGS-based molecular inversion probe (MIP) methodology) used by Schulte et al. [45] is much more sensitive, even leading to the reporting of changes present only in a single cell.

In this study a number of private genomic imbalances were observed but only a few recurrent CNAs. The most common numerical changes were trisomy 21 (4/13), monosomies 13, 18 and trisomy 19. Previously, trisomy 21 (also referred as 21 g gain) was reported in a notable portion of intracranial GCTs (64-76\%) $[44,45,50]$. That is in line with the observation of the higher risk of germ cell tumours in people with Down syndrome [21]. Among losses, chromosome 13 mono- somy (also referred as $13 q$ deletion) was reported in $36-53 \%$ of cases $[32,44,50]$. Here, of the Hh signalling-associated genes, ZIC2 gene, which modifies GLI action, is a potential candidate gene involved in oncogenesis. ZIC2, a member of the ZIC family of $\mathrm{C} 2 \mathrm{H} 2$-type zinc finger proteins, functions as a transcriptional repressor. The ZIC proteins cooperate with key transcriptional mediators of the Hedgehog, WNT and BMP pathways as well as with chromatin-modifying complexes. It is one of the most important factors affecting neural tube development, cell differentiation and proliferation. ZIC2 alterations have been connected to pathogenesis and progression of some tumours $[7,8,13,24,30]$. Another candidate gene, located at chromosome $13 q$ and hence notoriously affected by GCTs, is FGF9. It belongs to the fibroblast growth factor (FGF) gene family, encoding peptide regulatory factors that act through distinct tyrosine kinase receptors. The FGF family of genes is involved in numerous biologic processes occurring during embryogenesis and later life, comprising morphogenesis and possibly tumorigenesis [2,23].

The most common structural aberration, gain/ amplification of the fragment of the chromosome $12 p$, was present in $3 / 13$ samples. None of the other CNAs either observed in our study or reported previously was seen consistently $[19,23,36,45]$. In the previously published pilot study [25], we have identified only two recurrent aberrations, namely gains at 12p13.33p11.1 and 17q11.1q25.3. In the presented series of GCTs, extension of the studied group allowed for identification of these particular CNAs each in only one additional case. Gain/amplification at $12 p$ is the foremost CNA in GCTs of adulthood observed in up to $80 \%$ of cases of adult testicular GCTs, usually in the form of isochromosome $\mathrm{i}(12 \mathrm{p})$ [52]. Previous single reports demonstrated that the prevalence of $12 p$ gains in intracranial GCTs is lower than expected, namely 53\% [45], 33\% [44] and 59\% [50] of the studied cohorts. The frequency of $23 \%$ (3/13) described in our study is in line with these reports, pointing to the differences in carcinogenesis in adulthood testicular tumours and the extragonadal paediatric GCTs. Only Schulte et al. [45] reported a high (82\%) frequency of this aberration in GCTs by using the highly sensitive MIP methodology. The most promising candidate genes located at $12 \mathrm{p}$ are $K R A S$ and WNT5B, the latter ranked among Hh network partners. Actually, in our cohort a number of WNT gene family members were found to be affect- 
ed by CNAs in a subset of cases. The WNT gene family encodes secreted signalling proteins playing a role in several developmental processes. These proteins have also been implicated in carcinogenesis in a number of haematological and solid cancers through activation of the WNT- $\beta$-catenin-TCF signalling pathway [53].

In our series, the comprehensive, whole-genome evaluation of the Hh-related loci revealed, among the elements of the Hedgehog network, PTCH1, $G L I 2, I H H$ and ZIC2 genes to be affected most frequently in $4 / 13,2 / 13,2 / 13$ and $4 / 13$ cases, respectively. Moreover, 6 tumours had various copy gains or losses of several genes coding ligands, elements or regulators of the pathway (HHIP, GLI1, GLI3, DHH, $S H H, S M O, P T C H 2)$. Interestingly, four cases with different histology (\#1 - teratoma, \#2 - yolk sac, \#3 - germinoma, \#11 - germinoma) showed losses of pathway suppressors (PTCH1, PTCH2, HHIP, GLI3). In addition, two of them $(\# 2, \# 11)$ revealed parallel gains of pathway activators $(I H H, D H H, S H H, S M O$, GLI1, GLI2). Especially one germinoma revealed genetic changes suggesting that the Hh pathway alteration might have been involved in its carcinogenesis. This tumour showed loss of PTCH1-pathway suppressor gene, together with gains in many key genes of $\mathrm{Hh}$ signalling, including SMO and hedgehog ligand proteins. PTCH1, a member of the patched gene family, encodes the receptor for sonic, desert and Indian hedgehog ligands. Mutations of this gene have been found in esophageal squamous cell carcinoma, trichoepitheliomas, transitional cell carcinomas of the bladder as well as in holoprosencephaly $[26,35,47]$. The immunohistochemistry did not reveal differences in $\mathrm{SHH}$ and $\mathrm{PTCH} 1$ expression between two germinomas with different alterations in these genes. The expression pattern was dissimilar between germinomas and teratomas due to their histological composition.

Detection of chromosome copy number structural aberrations (CNAs) may lead to identification of candidate genes involved in processes related to tumorigenesis $[10,49]$. CNAs may affect the phenotype by altering gene dosage. Since the amount of protein synthesized is usually proportional to the number of gene copies present, genomic imbalances can lead to depletion or excess of particular proteins $[10,47,49]$. Because most embryonic developmental processes depend on carefully balanced levels of proteins, any imbalance may have severe consequenc- es [9]. Certain genes require two copies for normal expression levels to be produced, so deletion of one gene copy leads to haploinsufficiency and a pathogenic phenotype. Also, in that manner a recessive mutant allele present on the residual chromosome may become articulated $[10,49]$.

Our results show the presence of genomic alterations affecting, among others, also the loci of the genes related to the Hh signalling pathway in paediatric CNS GCTs. The limitation of the study is the histological heterogeneity of the examined series of these rare neoplasms. Although no recurrent CNAs were found to be distinctive for this group of tumours, the performed analysis of CNAs points at a few genes of the Hh pathway network as putative candidates involved in a subset of cases: PTCH1, ZIC2 and GL/13 in particular. Further analysis of Hedgehog pathway alterations are needed to disclose their biological significance.

\section{Ethical approval}

The study was approved by the Ethical Committee of the Medical University of Gdańsk, Poland.

\section{Disclosure}

This research was funded by Polish National Science Centre grant number: 2014/15/B/NZ4/04855.

The authors report no conflict of interest.

\section{References}

1. Aszterbaum M, Rothman A, Johnson RL, Fisher M, Xie J, Bonifas JM, Zhang X, Scott MP, Epstein Jr EH. Identification of mutations in the human PATCHED gene in sporadic basal cell carcinomas and in patients with the basal cell nevus syndrome. J Invest Dermatol 1998; 110: 885-888.

2. Beenken A, Mohammadi M. The FGF family: biology, pathophysiology and therapy. Nat Rev Drug Discov 2009; 8: 235-253.

3. Briscoe J, Therond PP. The mechanisms of Hedgehog signaling and its roles in development and disease. Nat Rev Mol Cell Biol 2013; 14: 416-429.

4. Bruggeman JW, Koster J, Lodder P, Repping S, Hamer G. Massive expression of germ cell-specific genes is a hallmark of cancer and a potential target for novel treatment development. Oncogene 2018; 37: 5694-5700.

5. Calaminus G, Frappaz D, Kortmann RD, Krefeld B, Saran F, Pietsch T, Vasiljevic A, Garre ML, Ricardi U, Mann JR, Göbel U, Alapetite C, Murray MJ, Nicholson JC. Outcome of patients with intracranial non-germinomatous germ cell tumors-lessons from the SIOPCNS-GCT-96 trial. Neuro Oncol 2017; 19: 1661-1672. 
6. Carballo GB, Honorato JR, de Lopes GPF, Spohr TCL de Se. A highlight on Sonic hedgehog pathway. Cell Commun Signal 2018; 16: 11.

7. Chan DW, Liu VW, Leung LY, Yao KM, Chan KK, Cheung AN, Ngan HY. Zic2 synergistically enhances Hedgehog signalling through nuclear retention of Gli1 in cervical cancer cells. J Pathol 2011: 225: 525-534.

8. Chervenak AP, Hakim IS, Barald KF. Spatiotemporal expression of Zic genes during vertebrate inner ear development. Dev Dyn 2013; 242: 897-908.

9. Cooper GM The cell: a molecular approach. $2^{\text {nd }}$ ed. Sunderland (MA): Sinauer Associates. Cell Proliferation in Development and Differentiation, 2000. Available from: https://wwwncbinImnihgov/books/NBK9906/

10. De Paz JF, Benito R, Bajo J, Rodríguez AE, Abáigar M. aCGH-MAS: analysis of aCGH by means of multiagent system. Biomed Res Int 2015; 194624

11. Deshpande G, Swanhart L, Chiang P, Schedl P. Hedgehog signaling in germ cell migration. Cell 2001; 106: 759-769.

12. Desphande G, Barr J, Gerlitz O, Lebedeva L, Shidlovskii Y, Schedl P. Cells on the move: Modulation of guidance cues during germ cell migration. Fly 2017; 11: 200-207.

13. Diamand KEM, Barratt KS, Arkell RM. Overview of Rodent Zic Genes. Adv Exp Med Biol 2018; 1046: 179-207.

14. Dufour C, Guerrini-Rousseau L, Grill J. Central nervous system germ cell tumors: an update. Curr Opin Oncol 2014; 26: 622626.

15. Echevarría ME, Fangusaro J, Goldman S. Pediatric central nervous system germ cell tumors: a review. Oncologist 2008; 13: 690-699.

16. Evangelista M, Tian H, de Sauvage FJ. The hedgehog signaling pathway in cancer. Clin Cancer Res 2008; 12: 5924-5928.

17. Gonnissen A, Isebaert S, Haustermans K. Targeting the Hedgehog signaling pathway in cancer: beyond Smoothened. Oncotarget 2015; 6: 13899-13913.

18. GröbnerSN, WorstBC, WeischenfeldtJ, Buchhalter I, KleinheinzK, Rudneva VA, Johann PD, Balasubramanian GP, Segura-Wang M, Brabetz S, Bender S, Hutter B, Sturm D, Pfaff E, Hübschmann D, Zipprich G, Heinold M, Eils J, Lawerenz C, Erkek S, Lambo S, Waszak S, Blattmann C, Borkhardt A, Kuhlen M, Eggert A, Fulda S, Gessler M, Wegert J, Kappler R, Baumhoer D, Burdach S, Kirschner-Schwabe R, Kontny U, Kulozik AE, Lohmann D, Hettmer S, Eckert C, Bielack S, Nathrath M, Niemeyer C, Richter GH, Schulte J, Siebert R, Westermann F, Molenaar JJ, Vassal G, Witt H, ICGC PedBrain-Seq Project, ICGC MMML-Seq Project, Burkhardt B, Kratz CP, Witt O, van Tilburg CM, Kramm CM, Fleischhack G, Dirksen U, Rutkowski S, Frühwald M, von Hoff K, Wolf S, Klingebiel T, Koscielniak E, Landgraf P, Koster J, Resnick AC, Zhang J, Liu Y, Zhou X, Waanders AJ, Zwijnenburg DA Raman P, Brors B, Weber UD, Northcott PA, Pajtler KW, Kool M, Piro RM, Korbel JO, Schlesner M, Eils R, Jones DTW, Lichter P, Chavez L, Zapatka M, Pfister SM. The landscape of genomic alterations across childhood cancers. Nature 2015; 555: 321-327.

19. Guo X, Wang X-F. Signaling cross-talk between TGF- $\beta / B M P$ and other pathways. Cell Res 2009; 19: 71-88.

20. Gupta S, Takebe N, LoRusso P. Targeting the Hedgehog pathway in cancer. Ther Adv Med Oncol 2010; 2: 237-250.
21. Hasle $\mathrm{H}$. Pattern of malignant disorders in individuals with Down's syndrome. Lancet Oncol 2001; 2: 429-436.

22. Huang X, Zhang R, Mao Y, Zhou LF, Zhang C. Recent advances in molecular biology and treatment strategies for intracranial germ cell tumors. World J Pediatr 2016; 12: 275.

23. Itoh N, Ornitz DM. Fibroblast growth factors: from molecular evolution to roles in development, metabolism and disease. J Biochem 2011; 149: 121-130.

24. Koyabu Y, Nakata K, Mizugishi K, Aruga J, Mikoshiba K. Physical and functional interactions between Zic and Gli proteins. J Biol Chem 2001; 276: 6889-6892.

25. Kuleszo D, Koczkowska M, Lipska-Ziętkiewicz BS, Grajkowska W, Adamkiewicz-Drożyńska E, Dembowska-Bagińska B, Ciołkowski M, lżycka-Świeszewska E. Comparative genomic analysis of intracranial germ cell tumors - the preliminary study focused on Sonic Hedgehog signaling pathway. Contemp Oncol 2017; 21: $279-284$.

26. Laukkanen M, Castellone M. Hijacking the Hedgehog pathway in cancer therapy. Anti-Cancer Agents Med Chem 2016; 16.

27. Lum L, Beachy PA. The Hedgehog response network: sensors, switches, and routers. Science 2004; 304: 1755-1759.

28. MacDonald JR, Ziman R, Yuen RK, Feuk L, Scherer SW. The database of genomic variants: a curated collection of structural variation in the human genome. Nucleic Acids Res 2014; 42 (Database issue): D986-992.

29. Macdonald TJ. Hedgehog Pathway in Pediatric Cancers: They're Not Just for Brain Tumors Anymore. Am Soc Clin Oncol Educ Book 2012; 605-609.

30. Marchini S, Poynor E, Barakat RR, Clivio L, Cinquini M, Fruscio R, Porcu L, Bussani C, D'Incalci M, Erba E, Romano M, Cattoretti G, Katsaros D, Koff A, Luzzatto $L$. The zinc finger gene ZIC2 has features of an oncogene and its overexpression correlates strongly with the clinical course of epithelial ovarian cancer. Clin Cancer Res 2012; 18: 4313-4324.

31. Miller SA, Dykes DD, Polesky HF. A simple salting out procedure for extracting DNA from human nucleated cells. Nucleic Acids Res 1988; 16: 1215.

32. Okada Y, Nishikawa R, Matsutani M, Louis DN. Hypomethylated $X$ chromosome gain and rare isochromosome $12 p$ in diverse intracranial germ cell tumors. J Neuropathol Exp Neurol 2002; 61: 531-538.

33. Online Mendelian Inheritance in Man, OMIM ${ }^{\circledast}$ McKusick-Nathans Institute of Genetic Medicine, Johns Hopkins University (Baltimore, MD). Available online: https://omimorg (accessed on 14.08.2018).

34. Oosterhuis WJ, Looijenga LH. Germ cell tumors from a developmental perspective: cells of origin, pathogenesis, and molecular biology (emerging patterns) In: Nogales FF, Jimenez RE (Eds.). Pathology and Biology of Human Germ Cell Tumors. Springer-Verlag GmbH, Berlin, Germany 2017, pp. 11-21.

35. Pak E, Segal RA. Hedgehog signal transduction: key players, oncogenic drivers, and cancer therapy. Dev Cell 2016; 38: 333-344.

36. Parascandolo A, Laukkanen MO, De Rosa N, Ugolini C, Cantisani MC, Cirafici AM, Basolo F, Santoro M, Castellone MD. A dual mechanism of activation of the Sonic Hedgehog pathway in anaplastic thyroid cancer: crosstalk with RAS-BRAF-MEK path- 
way and ligand secretion by tumor stroma. Oncotarget 2018; 9: 4496-4510.

37. Petrova R, Joyner AL. Roles for Hedgehog signaling in adult organ homeostasis and repair. Development 2014; 141: 3445 3457.

38. Pierce JL, Frazier AL, Amatruda JF. Pediatric germ cell tumors: a developmental perspective. Adv Urol 2018; 2018: 9059382.

39. Plant AS, Chi SN, Frazier L. Pediatric malignant germ cell tumors: A comparison of the neuro oncology and solid tumor experience. Pediatr Blood Cancer 2016; 63: 2086-2095.

40. Pócza T, Krenács T, Turányi E, Csáthy J, Jakab Z, Hauser P. High expression of DNA methyltransferases in primary human medulloblastoma. Folia Neuropathol 2016; 54: 105-113.

41. Renault AD, Ricardo S, Kunwar PS, Santos A, Starz-Gaiano M, Stein J, Lehmann R. Hedgehog does not guide migrating Drosophila germ cells. Dev Biology 2009; 328: 355-362.

42. Richardson BE, Lehmann R. Mechanisms guiding primordial germ cell migration: strategies from different organisms. Nat Rev Mol Cell Biol 2010; 11: 37-49.

43. Ruiz I Altaba A, Stecca B, Sánchez P. Hedgehog-Gli signaling in brain tumors: stem cells and paradevelopmental programs in cancer. Cancer Lett 2004; 20: 145-157.

44. Schneider DT, Zahn S, Sievers S, Alemazkour K, Reifenberger G, Wiestler OD, Calaminus G, Göbel U, Perlman EJ. Molecular genetic analysis of central nervous system germ cell tumors with comparative genomic hybridization. Mod Pathol 2006; 19: 864-873.

45. Schulte SL, Waha A, Steiger B, Denkhaus D, Dörner E, Calaminus G, Leuschner I, Pietsch T. CNS germinomas are characterized by global demethylation, chromosomal instability and mutational activation of the Kit-, Ras/Raf/Erk- and Akt-pathways. Oncotarget 2016; 7: 55026-55042.

46. Shen H, Shih J, Hollern DP, Wang L, Bowlby R, Tickoo SK, Thors son V, Mungall AJ, Newton Y, Hegde AM, Armenia J, Sánchez-Vega F, Pluta J, Pyle LC, Mehra R, Reuter VE, Godoy G, Jones J, Shelley CS, Feldman DR, Vidal DO, Lessel D, Kulis T, Cárcano FM, Leraas KM, Lichtenberg TM, Brooks D, Cherniack AD, Cho J, Heiman DI, Kasaian K, Liu M, Noble MS, Xi L, Zhang H, Zhou W, ZenKlusen JC, Hutter CM, Felau I, Zhang J, Schultz N, Getz G, Meyerson M, Stuart JM, Cancer Genome Atlas Research Network, Akbani R, Wheeler DA, Laird PW, Nathan-son KL, Cortessis VK, Hoadley KA. Integrated molecular characterization of testicular germ cell tumors. Cell Rep 2018; 23: 3392 3406.

47. Skoda AM, Simovic D, Karin V, Kardum V, Vranic S, Serman L. The role of the Hedgehog signaling pathway in cancer: A comprehensive review. Bosnian J Basic Med Sci 2018; 18: 8-20.

48. Tan C, Scotting PJ. Stem cell research points the way to the cell of origin for intracranial germ cell tumours. J Pathol 2013; 229: 4-11.

49. Tang YC, Amon A. Gene copy number alterations: A cost-benefit analysis. Cell 2013; 152: 394-405.

50. Terashima K, Yu A, Chow W, Hsu WC, Chen P, Wong S, Hung YS, Suzuki T, Nishikawa R, Matsutani M, Nakamura H, Ng HK, Allen JC, Aldape KD, Su JM, Adesina AM, Leung HC, Man TK, Lau CC. Genome-wide analysis of DNA copy number alterations and loss of heterozygosity in intracranial germ cell tumors. Pediatr Blood Cancer 2014; 61: 593-600.
51. Varjosalo M, Taipale J. Hedgehog: functions and mechanisms. Genes Dev 2008; 22: 2454-2472.

52. Wang HW, Wu YH, Hsieh JY, Liang ML, Chao ME, Liu DJ, Hsu MT, Wong TT. Pediatric primary central nervous system germ cell tumors of different prognosis groups show characteristic miRNome traits and chromosome copy number variations. BMC Genomics 2010; 11: 132.

53. Zhan T, Rindtorff N, Boutros M. Wnt signaling in cancer. Oncogene 2017; 36: 1461-1473. 